\title{
CYTOKERATIN 19 IMMUNOREACTIVITY IN PAPILLARY THYROID CARCINOMA
}

\author{
Chithira Balakrishnan ${ }^{1}$, Sheela Varghese'2, Sankar $S^{3}$ \\ ${ }^{1}$ Senior Resident, Department of Pathology, Government Medical College, Kottayam. \\ ${ }^{2}$ Associate Professor, Department of Pathology, Government Medical College, Kottayam. \\ ${ }^{3}$ Professor and HOD, Department of Pathology, Government Medical College, Kottayam.
}

\section{ABSTRACT}

\section{BACKGROUND}

Papillary carcinoma is the most common malignant lesion of the thyroid gland. Diagnostic difficulties can be solved by the use of immunohistochemical markers. Several studies have shown conflicting results regarding the usefulness of cytokeratin 19 as a diagnostic marker in papillary carcinoma. This study was conducted to assess the role of cytokeratin 19 as a possible diagnostic marker in papillary thyroid carcinoma. The objective of the present study was to evaluate the expression of cytokeratin 19 in papillary thyroid carcinoma and also to describe the patterns of Papillary thyroid carcinoma in Histopathology, Radiology, Cytology and clinical presentation.

\section{MATERIALS AND METHODS}

A descriptive study was conducted to assess Cytokeratin 19 immunoreactivity in papillary thyroid carcinoma specimens received in the Department of Pathology, Government Medical College, Kottayam during a period of 18 months (January 2015-June 2016). Statistical analysis done with available software.

\section{RESULTS}

Among the 32 cases of Papillary carcinoma studied, 21 (66\%) were conventional type, 7 (22\%) were Follicular variants and 4 $(12 \%)$ were papillary microcarcinomas. Diffuse Cytokeratin 19 immunoreactivity was found in 93.75\% cases. Diffuse Cytokeratin 19 staining seen in $95 \%$ cases of conventional papillary carcinoma, $85 \%$ of follicular variants of papillary carcinoma and $100 \%$ of papillary microcarcinoma. Analysis of clinical presentation of these cases revealed only previously described findings similar to previously described studies. However, the cytological findings showed a tendency to give a diagnosis of benign follicular nodule in $53 \%$ of cases.

\section{CONCLUSION}

Cytokeratin 19 showed consistent diffuse positivity in histopathology of Papillary thyroid carcinoma and its variants, which indicate the role of cytokeratin 19 as a diagnostic marker. The findings are comparable to other Indian studies on this subject.

\section{KEYWORDS}

Cytokeratin 19; Papillary Thyroid Carcinoma; Immunohistochemistry.

HOW TO CITE THIS ARTICLE: Balakrishnan C, Varghese S, Sankar S. Cytokeratin 19 immunoreactivity in papillary thyroid carcinoma. J. Evolution Med. Dent. Sci. 2017;6(77):5474-5477, DOI: 10.14260/Jemds/2017/1188

\section{BACKGROUND \\ Papillary carcinoma is the most common malignant lesion of the thyroid gland. Despite the propensity for lymphatic dissemination to cervical lymph nodes, the majority of patients with these tumours, if appropriately treated, have an excellent longterm prognosis. Appropriate treatment then rests on the ability of the pathologist to render an accurate diagnosis. \\ The diagnosis of papillary carcinoma is based on nuclear features such as nuclear clearing, overlapping, grooving and pseudoinclusions. However, identification of these features remains, at times, difficult, because of its focal presence and thus distinction of papillary carcinoma from other thyroid lesions may not be possible.}

Financial or Other, Competing Interest: None.

Submission 13-08-2017, Peer Review 14-09-2017,

Acceptance 19-09-2017, Published 25-09-2017.

Corresponding Author:

Dr. Sheela Varghese,

Associate Professor,

Department of Pathology,

Government Medical College,

Kottayam, Kerala.

E-mail: drsheelavarghese@gmail.com

DOI: $10.14260 /$ jemds $/ 2017 / 1188$
Several variants of papillary carcinoma has been described, among these follicular variants of papillary carcinoma is the most common one which is characterised by an almost exclusive follicular growth pattern and a set of nuclear features identical to those of usual type of papillary carcinoma. Diagnostic dilemma may arise when an encapsulated nodule with follicular pattern exhibits clear nuclei with grooves or darkly staining colloid, and distinguishing follicular adenoma from follicular variant of papillary carcinoma becomes difficult. The distinction between these different lesions is important because their management and prognosis are different.

In an attempt to solve this common diagnostic difficulty, many immunohistochemical markers have been evaluated for their potential in distinguishing papillary thyroid carcinoma from other follicular lesions. The main ones include cytokeratin 19, Galectin 3 and HBME 1.

Several studies have shown conflicting results regarding the usefulness of cytokeratin 19 as a diagnostic marker in papillary carcinoma. This study was conducted to assess the role of cytokeratin 19 as a possible diagnostic marker in papillary thyroid carcinoma. 


\section{MATERIALS AND METHODS}

Type of Study

Descriptive study.

\section{Study Period}

18 months (January 2015-June 2016).

\section{Study Setting}

Department of Pathology, Government Medical College, Kottayam

\section{Sample size}

32

Sample size $\mathrm{N}=4 \mathrm{pq} / \mathrm{d}^{2}$

$\mathrm{p}=$ prevalence $/$ proportion in previous study

$\mathrm{q}=100-\mathrm{p}$

$\mathrm{d}=$ precision/allowable error

Proportion of papillary thyroid carcinoma cases showed cytokeratin 19 positivity in previous study $=98 \%$.

So, $\mathrm{p}=98, \mathrm{q}=100-98=2$

Taking allowable error as $5 \%$.

Sample size, $\mathrm{N}=4 \mathrm{pq}=4 \times 98 \times 2=31.36$

$d^{2} 5 \times 5$

Taking sample size as 32 .

\section{Inclusion Criteria}

Histopathologically diagnosed cases of papillary thyroid carcinoma including variants were included.

\section{Exclusion Criteria}

Cases without proper data, cases with indefinite diagnosis, recurrent/treated cases of papillary thyroid carcinoma were excluded.

\section{Study Procedure}

Clinical details of each case were recorded first along with radiological and cytological results. Gross examination of the specimen was carried out. Tumour size was measured in three dimensions and the largest dimension was taken into account.

Appropriate bits of tissues representative of areas to be studied were taken. All specimens were fixed in formalin and embedded in paraffin. 4 microns thick sections was stained with $\mathrm{H}$ \& $\mathrm{E}$ for routine histological examination. Immunohistochemical staining was performed using rabbit monoclonal cytokeratin 19 antibody. Immunoreactivity is assessed according to the distribution and intensity of staining. Positive control used was human colonic tissue. Negative control made by omitting primary antibody. CK 19 gives membrane positivity with or without cytoplasmic positivity in papillary thyroid carcinoma.

A semiquantitative assessment of immunohistochemical scoring was performed. Immunoreactivity was considered positive if more than $10 \%$ of follicular epithelial cells stained. Immunoreactivity was scored as negative, focally positive (+: less than $25 \%)$, positive $(++: 25-50 \%)$ or diffusely positive $(+++$ : more than $50 \%)$ based on the extent of reaction.

\section{Data Management and Analysis}

The data was entered in Microsoft excel and further statistical analysis was done using available institutional software.

\section{RESULTS}

The mean age of papillary carcinoma is found to be 43.8 years with Female: male ratio of 5.4:1.
None of the cases had past history of radiation exposure/cancer.

9 cases (28\%) gave history of symptoms of compression (dyspnoea/dysphagia/hoarseness of voice/pain).

21 cases $(66 \%)$ had palpable thyroid swelling only. 2 cases $(6 \%)$ had cervical lymphadenopathy only. 9 cases (28\%) had both thyroid swelling and lymphadenopathy.

Ultrasound scans have given diagnosis of malignancy in $59 \%$ cases only. Only $25 \%$ cases were diagnosed as papillary carcinoma on FNAC.

Out of the 32 cases of PTC studied, 21 (66\%) were conventional PTCs, 7 (22\%) were Follicular variants and 4 $(12 \%)$ were papillary microcarcinomas.

Nuclear crowding, overlapping and grooving were present in $91 \%$ of cases.

Intranuclear inclusions were seen only in $16 \%$ of cases. Nuclear clearing was seen in all cases. Lymphocytic thyroiditis was present in $50 \%$ of cases. ?10 cases (31\%) had Lymph node metastasis.

Diffuse Cytokeratin 19 immunoreactivity was found in 93.75\% cases of PTC. Diffuse CK 19 staining seen in 95\% cases conventional PTC, $85 \%$ of follicular variants of PTC and $100 \%$ of papillary microcarcinomas.

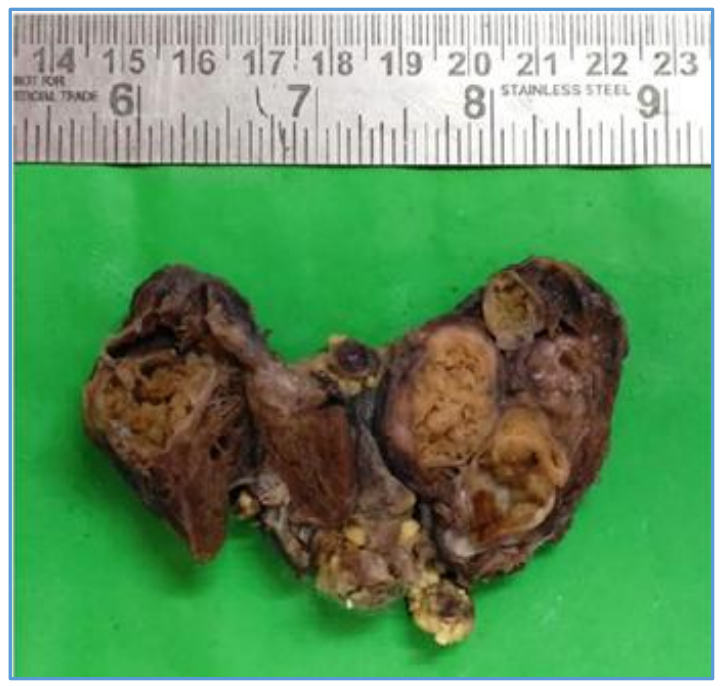

Figure 1. Gross Photograph of Conventional Papillary Thyroid Carcinoma, Cut Section is Whitish and Granular

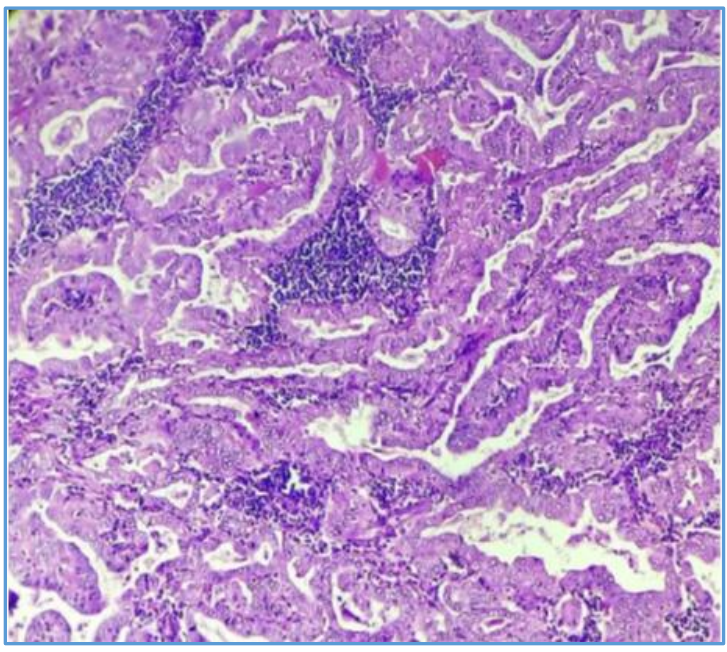

Figure 2. Photomicrograph (20x) of Conventional PTC (H\&E Stain) 


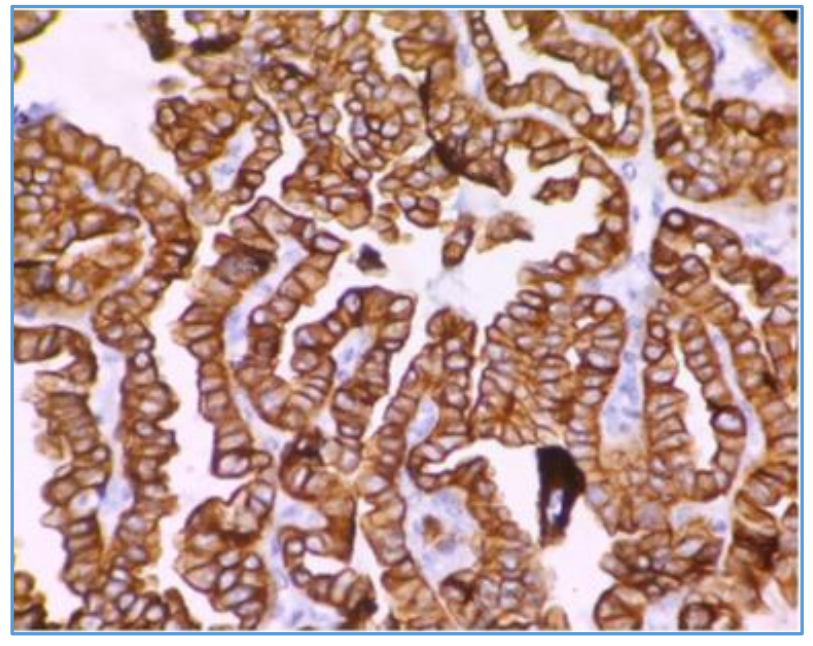

Figure 3. Photomicrograph (40x) of IHC- CK 19 Positive Staining - Conventional PTC

\section{DISCUSSION}

The diagnosis of papillary carcinoma is based on nuclear features. Some benign lesions like nodular goitre, thyroiditis, thyrotoxicosis, adenomas may show papillary architecture and PTC like nuclear features. In such situations, we can distinguish papillary carcinoma from other lesions by doing immunohistochemistry. Several studies have shown conflicting results regarding the usefulness of cytokeratin 19 as a diagnostic marker in papillary carcinoma. This study was conducted to investigate the role of cytokeratin 19 as a possible diagnostic marker in papillary thyroid carcinoma.

The present study was conducted on 32 cases of papillary thyroid carcinoma specimens received in department of Pathology, Govt. Medical College, Kottayam between January 2015 and July 2016.

The mean age of papillary carcinoma in the present study is 43.8 years, which is comparable with studies done by Sahoo et al in 35 cases of PTC in New York, which showed a mean age of 46 years. ${ }^{1}$ Studies by Debdas et al showed a mean age of 34.5 years. ${ }^{2}$

Female: male ratio is 5.4:1 in the present study. According to Negri et al, PTC was about four-times more common in females than in males. ${ }^{3}$ In Debdas Bose et al studies, the female: male ratio, in cases of PTC, was 6.3:1.2

Considering the history of patients, 9 cases (28\%) gave history of symptoms of tumour compression in the forms of dyspnoea/dysphagia/hoarseness of voice/pain.

Patients attended surgery OPD due to these symptoms rather than their thyroid swelling. Majority of the cases got medical attention within 2 years of onset of symptoms.

Previous Studies show that Individuals exposed to external or internal radiation have increased biological sensitivity to PTC. But none of the cases had past history of radiation exposure/cancer in the present study.

Considering clinical presentation, 21 cases (66\%) had palpable thyroid swelling only. 2 cases $(6 \%)$ had cervical lymphadenopathy only. 9 cases (28\%) had both thyroid swelling and lymphadenopathy. Studies by Carcangiu et al showed that disease was localised to the thyroid gland in $67 \%$ of cases, thyroid and lymph nodes in $13 \%$, and lymph nodes alone in 20\%. Most cases of PTC present as asymptomatic thyroid nodules, but first manifestation may be an enlarged cervical lymph node.
Analysis of thyroid function tests results showed $81 \%$ of cases were euthyroid, 13\% were hypothyroid and 6\% were hyperthyroid. According to DeLellis et al, thyroid carcinomas rarely interfere with functional capacity of thyroid gland. ${ }^{4}$

The only diagnostic imaging modality used in all cases was ultrasound of neck/thyroid. $59 \%$ of cases had an ultrasound diagnosis of thyroid malignancy, $41 \%$ cases were diagnosed as benign on ultrasonogram. Hence, Ultrasound scan is found to be useful to find the size of a thyroid lesion, solid and cystic nature, guiding the performance of FNA.

\section{FNAC Diagnosis}

Only $25 \%$ cases were diagnosed as papillary carcinoma, $16 \%$ cases were diagnosed as suspicious of papillary carcinoma and $53 \%$ cases were diagnosed as benign follicular nodule on FNAC. We could not review the FNAC slides.

False negative diagnosis occurs as a result of inadequate sampling and the presence of infrequent nuclear features of PTC. Cystic PTC is another diagnostic challenge and can be missed on FNA.

Awareness of pathologists regarding these pitfalls of FNAC and meticulous examination of smears with strict adherence to the diagnostic criteria of PTC can reduce false positive and false negative diagnoses and thus provide better patient care. ${ }^{5}$

Out of the 32 cases of PTC studied, 21 (66\%) were conventional PTCs, 7 (22\%) were follicular variants and 4 (12\%) were papillary microcarcinomas. In Debdas Bose et al study, 22 cases were PTC, of which 8 (37\%) cases were follicular variants.

PTC, a single case $(4 \%)$ was diffuse sclerosing variant and the remaining 13 (59\%) were conventional PTC.2 Alshenawy et al studied 22 cases of PTC in Tanta, Egypt, 14 (64\%) cases were conventional PTCs and $8(36 \%)$ were follicular variants. ${ }^{6}$

\begin{tabular}{|c|c|c|c|c|}
\hline & $\begin{array}{c}\text { Conventional } \\
\text { PTC }\end{array}$ & $\begin{array}{c}\text { Follicular } \\
\text { Variant }\end{array}$ & $\begin{array}{c}\text { Micro } \\
\text { carcinoma }\end{array}$ & $\begin{array}{c}\text { Diffuse } \\
\text { Sclerosing } \\
\text { Variant }\end{array}$ \\
\hline $\begin{array}{c}\text { Present } \\
\text { Day }\end{array}$ & $66 \%$ & $22 \%$ & $12 \%$ & 0 \\
\hline $\begin{array}{c}\text { Debdas } \\
\text { et al }\end{array}$ & $59 \%$ & $37 \%$ & 0 & $4 \%$ \\
\hline $\begin{array}{c}\text { Alshenawy } \\
\text { et al }\end{array}$ & $64 \%$ & $36 \%$ & 0 & 0 \\
\hline Table 1. Comparison of Histological Variants of PTC \\
Studied with other Studies \\
\hline
\end{tabular}

Considering nuclear features, nuclear crowding, overlapping and grooving present in $91 \%$ of cases. Intranuclear inclusions seen only in $16 \%$ of cases. Nuclear clearing seen in all cases. But none of the nuclear features is pathognomonic of PTC.

Lymphocytic thyroiditis present in $50 \%$ of cases. According to Thamimi et al 58\% of PTC cases showed lymphocytic thyroiditis. An immunologic mechanism involved in the pathogenesis of PTC may stimulate lymphocytic infiltration in the thyroid tissue. ${ }^{7}$

Diffuse Cytokeratin 19 immunoreactivity was found in 30 (93.75\%) cases of PTC in the present study. Studies by Debdas et al $(\mathrm{N}=22$, in West Bengal, India) showed CK 19 expression in $100 \%$ cases of PTC. ${ }^{2}$ Alshenawy et al $(\mathrm{N}=22$, in Tanta, Egypt) study showed CK 19 immunoreactivity in 100\% 
cases of PTC. ${ }^{6}$ Saleh et al (N=32, in Michigan, USA) study showed CK 19 immunoreactivity in 85 \% cases of PTC. 8

\begin{tabular}{|c|c|}
\hline Present Study & $93.75 \%$ \\
\hline Debdas et al & $100 \%$ \\
\hline Alshenawy et al & $100 \%$ \\
\hline Saleh et al & $85 \%$ \\
\hline Table 2. Comparison of CK 19 Immunoreactivity in PTC \\
with Other Studies \\
\hline
\end{tabular}

In the present study, diffuse CK 19 staining seen in 95\% cases of conventional PTC, 85\% of follicular variants of PTC and $100 \%$ of papillary microcarcinomas.

According to Sahoo et al ( $\mathrm{N}=35$, in New York), 93\% of follicular variants of PTC and all cases of conventional PTC showed diffuse positivity for CK19.1 Studies by Debdas et al ( $\mathrm{N}=22$, in West Bengal, India) showed diffuse CK 19 staining in $84.5 \%$ of conventional PTC and $62.5 \%$ of follicular variants of PTC. ${ }^{2}$

\begin{tabular}{|c|c|c|c|}
\hline & Conventional & $\begin{array}{c}\text { Follicular } \\
\text { Variant }\end{array}$ & $\begin{array}{c}\text { Micro- } \\
\text { carcinoma }\end{array}$ \\
\hline Present Study & $95 \%$ & $85 \%$ & $100 \%$ \\
\hline Sahoo et al & $100 \%$ & $93 \%$ & - \\
\hline Debdas et al & $84.5 \%$ & $62.5 \%$ & - \\
\hline $\begin{array}{r}\text { Table 3. Comparison of Diffuse CK 19 Positivity among } \\
\text { Various Histological Types with other Studies }\end{array}$ \\
\hline
\end{tabular}

\section{CONCLUSION}

Based on the present study, it is concluded that Cytokeratin 19 can be used as a good diagnostic marker for papillary thyroid carcinoma.

\section{ACKNOWLEDGEMENTS}

I express my heartfelt thanks \& gratitude to Dr Sankar S, Head of the Department, Department of Pathology, Government Medical College, Kottayam and my guide $\mathrm{Dr}$ Sheela Varghese, Associate Professor, Department of Pathology, Government Medical College, Kottayam for their passion, dedication and help.

\section{REFERENCES}

[1] Sahoo S, Hoda SA, Rosai J, et al. Cytokeratin 19 immunoreactivity in the diagnosis of papillary thyroid carcinoma: a note of caution. Am J Clin Pathol 2001;116(5):696-702.

[2] Bose D, Das RN, Chatterjee U, et al. Cytokeratin 19 immunoreactivity in the diagnosis of papillary thyroid carcinoma. Indian J Med Paediatr Oncol 2012;33(2):107-11.

[3] Negri E, Dal Maso L, Ron E, et al. A pooled analysis of case-control studies of thyroid cancer. II. Menstrual and reproductive factors. Cancer Causes Control 1999;10(2):143-55.

[4] DeLellis RA, Lloyd RV, Heitz PU, et al. Pathology and genetics of tumours of endocrine organs. In: Kleihues P, Sobrin LH, (eds). World Health Organization. Classification of tumours. Lyon: IARC Press, 2004:4966.

[5] Sharma C. An analysis of trends of incidence and cytohistological correlation of papillary carcinoma of the thyroid gland with evaluation of discordant cases. J Cytol 2016;33(4):192-8.

[6] Alshenawy HA. Utility of immunohistochemical markers in differential diagnosis of follicular cell derived thyroid lesions. Journal of Microscopy and Ultrastructure 2014;2(3):127-36.

[7] Tamimi DM. The association between chronic lymphocytic thyroiditis and thyroid tumors. Int J Surg Pathol 2002;10(2):141-6.

[8] Saleh HA, Jin B, Barnwell J, et al. Utility of IHC markers in differentiating benign from malignant follicular derived thyroid nodules. Diagn Pathol 2010;5:9. 